\title{
The effect of deformability on the microscale flow behavior of red blood cell suspensions
}

Cite as: Phys. Fluids 31, 091903 (2019); https://doi.org/10.1063/1.5111189

Submitted: 25 May 2019 . Accepted: 02 September 2019 . Published Online: 25 September 2019

Andreas Passos, Joseph M. Sherwood (D), Efstathios Kaliviotis (D), Rupesh Agrawal (D), Carlos Pavesio, and Stavroula Balabani (D)

\section{COLLECTIONS}

Paper published as part of the special topic on Selected Articles from the 2018 Micro and Nano Flows Conference Note: This paper is part of the Special Issue from the 2018 Micro and Nano Flows Conference.
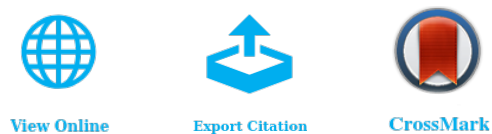

\section{ARTICLES YOU MAY BE INTERESTED IN}

Particle and rigidized red blood cell concentration distributions in microchannel flows

Physics of Fluids 31, 082006 (2019); https://doi.org/10.1063/1.5111201

Simulation of blood flow past a distal arteriovenous-graft anastomosis at low Reynolds numbers

Physics of Fluids 31, 091902 (2019); https://doi.org/10.1063/1.5099635

Directionally controlled open channel microfluidics

Physics of Fluids 31, 092003 (2019); https://doi.org/10.1063/1.5118728

\section{AIP Author Services English Language Editing}

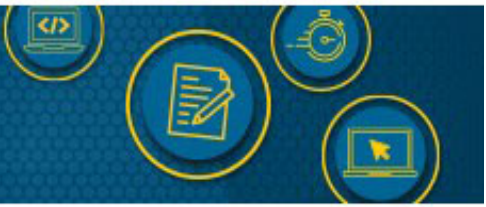




\title{
The effect of deformability on the microscale flow behavior of red blood cell suspensions
}

\author{
Cite as: Phys. Fluids 31, 091903 (2019); doi: 10.1063/1.5111189 \\ Submitted: 25 May 2019 - Accepted: 2 September 2019 • \\ Published Online: 25 September 2019
}

\begin{abstract}
Andreas Passos, ${ }^{1}$ Joseph M. Sherwood, ${ }^{2}$ (D) Efstathios Kaliviotis, ${ }^{3}$ (D) Rupesh Agrawal, ${ }^{4}$ Carlos Pavesio, ${ }^{5,6}$ and Stavroula Balabani ${ }^{1,2}$

AFFILIATIONS

'Department of Mechanical Engineering, University College London, London, United Kingdom

${ }^{2}$ Department of Bioengineering, Imperial College London, London, United Kingdom

${ }^{3}$ Department of Mechanical Engineering and Materials Science and Engineering, Cyprus University of Technology, Limassol, Cyprus

${ }^{4}$ National Healthcare Group Eye Institute, Tan Tock Seng Hospital, Singapore

${ }^{5}$ Moorfields Eye Hospital, London, United Kingdom

${ }^{6}$ Biomedical Research Centre, Institute of Ophthalmology, University College London, London, United Kingdom
\end{abstract}

Note: This paper is part of the Special Issue from the 2018 Micro and Nano Flows Conference.

a) s.balabani@ucl.ac.uk

\begin{abstract}
Red blood cell (RBC) deformability is important for tissue perfusion and a key determinant of blood rheology. Diseases such as diabetes, sickle cell anemia, and malaria, as well as prolonged storage, may affect the mechanical properties of RBCs altering their hemodynamic behavior and leading to microvascular complications. However, the exact role of RBC deformability on microscale blood flow is not fully understood. In the present study, we extend our previous work on healthy RBC flows in bifurcating microchannels [Sherwood et al., "Viscosity and velocity distributions of aggregating and non-aggregating blood in a bifurcating microchannel," Biomech. Model. Mechanobiol. 13, 259-273 (2014); Sherwood et al., "Spatial distributions of red blood cells significantly alter local hemodynamics," PLoS One 9, e100473 (2014); and Kaliviotis et al., "Local viscosity distribution in bifurcating microfluidic blood flows," Phys. Fluids 30, 030706 (2018)] to quantify the effects of impaired RBC deformability on the velocity and hematocrit distributions in microscale blood flows. Suspensions of healthy and glutaraldehyde hardened RBCs perfused through straight microchannels at various hematocrits and flow rates were imaged, and velocity and hematocrit distributions were determined simultaneously using micro-Particle Image Velocimetry and light transmission methods, respectively. At low feed hematocrits, hardened RBCs were more dispersed compared to healthy ones, consistent with decreased migration of stiffer cells. At high hematocrit, the loss of deformability was found to decrease the bluntness of velocity profiles, implying a reduction in shear thinning behavior. The hematocrit bluntness also decreased with hardening of the cells, implying an inversion of the correlation between velocity and hematocrit bluntness with loss of deformability. The study illustrates the complex interplay of various mechanisms affecting confined RBC suspension flows and the impact of both deformability and feed hematocrit on the resulting microstructure.
\end{abstract}

Published under license by AIP Publishing. https://doi.org/10.1063/1.5111189

\section{INTRODUCTION}

Microvascular flow plays a vital role in regulating physiological functions, such as vascular resistance, and maintaining organ health. A large number of pathologies alter these flows, leading to complications and posing a risk to human health. Understanding the hemodynamics and rheology of blood flow in the microvasculature is thus of great importance in health and disease.
Red blood cells (RBCs) or erythrocytes are the most abundant cells in blood, with a volume fraction of $45 \%$ (known as hematocrit); hence, blood can be considered as a soft particle suspension, i.e., comprising deformable cells suspended in a continuous phase, the plasma (which is typically assumed Newtonian). As such, blood exhibits shear-thinning behavior, determined to a great extent by the deformability and aggregation of RBCs. The ability of RBCs to deform is key to their function of nutrient and waste transport as 
it allows them to flow through microvessels with dimensions comparable to or smaller than their size. Pathologies such as sickle cell anemia, malaria, or diabetes are associated with both less deformable RBCs and microangiopathies, such as ischemia and vaso-occlusion. ${ }^{4}$ Decreased RBC deformability has been reported in malaria, ${ }^{5}$ sickle cell anemia, ${ }^{6}$ and other metabolic inflammatory or vascular diseases such as diabetes, Alzheimer, and stroke. . $^{7-10}$

The role of RBC deformability on blood rheology has been the subject of numerous studies. Early work by Chien ${ }^{11-13}$ revealed that hardened RBC suspensions exhibit almost Newtonian behavior and higher viscosity compared to washed normal RBCs. The increase in effective viscosity has been attributed to an increase in tumbling motion of hardened cells, most notably glutaraldehyde treated ones. ${ }^{14}$ More recently, Lanotte et al. ${ }^{15}$ confirmed the near Newtonian behavior of hardened RBCs and provided further evidence that $\mathrm{RBC}$ deformability as well as changes in RBC dynamic morphologies and motion during shearing account for the shear thinning blood behavior in microcirculation. An increase in viscosity and loss of shear thinning behavior have also been demonstrated in rheological studies of sickle RBC suspensions ${ }^{6,16}$ in which RBC deformability is reduced.

The increased viscosity of less deformable RBCs has an impact on flow resistance and hence microvascular flow and perfusion. In vivo animal studies have shown that reduced RBC deformability increases vascular resistance ${ }^{13,17}$ and affects oxygen delivery and tissue perfusion in a manner comparable to extreme anemia. ${ }^{18}$ In vitro measurements in artificial microvascular networks have also demonstrated higher flow resistance of hardened or aged RBCs compared to healthy ones. ${ }^{19,20}$ Similarly, decreased hydrodynamic conductance has been found in flows of sickle (less deformable) RBCs, serving thus as an effective biomarker ${ }^{21}$ to stratify sickle cell patients.

The deformability, along with the aggregation tendency of RBCs, forms the basis for many biophysical phenomena in microcirculatory flows. Deformability allows a symmetry breaking in the low Reynolds number Stokes flows encountered in the microcirculation (which prohibit migration of rigid particles), inducing a wall lift force on RBCs. This force together with the lift generated by the shear gradients in inhomogeneous flows in the microcirculation drives deformable RBCs away from the wall and toward the microvessel centerline. Lateral migration of RBCs leads to heterogeneous distributions throughout the microvasculature and to the Fåhraeus and Fåhraeus-Lindqvist effects. Both single RBC motion and migration dynamics are strongly influenced by the mechanical properties of RBCs, and rigidified cells exhibit tumbling motion ${ }^{14,22}$ and reduced migration rates. ${ }^{23,24}$ At physiological hematocrits, RBC migration is counterbalanced by cell hydrodynamic interactions (collectively termed shear induced diffusion), ${ }^{25}$ and the two phenomena combined give rise to heterogeneous cell distributions in the microcirculation.

A number of in silico studies have investigated the role of deformability on microscale blood flow, for example, by Fedosov et al., ${ }^{26}$ Zhang et al., ${ }^{27}$ and Bagchi et al., ${ }^{28}$ showing increased dispersion of less deformable RBCs due to their tumbling motion and a reduction in the cell depletion layers. However, there are few experimental studies on the effects of reduced RBC deformability on velocity and hematocrit distributions for physiological hematocrits, with published studies dealing mostly with dilute RBC suspensions ${ }^{29,30}$ or margination of hardened RBCs. ${ }^{31,32}$ Experiments with dilute suspensions of hardened RBCs have been shown to be more widely distributed in a Poiseuille flow compared to deformable $\mathrm{RBCs}^{29}$ in agreement with in silico findings. An inversion of hematocrit partitioning in bifurcations has also been reported by Shen et al. ${ }^{30}$ for dilute suspensions with high viscosity contrast highlighting the impact of altered RBC deformability on hematocrit distributions in the microvasculature. A small amount of rigidified RBCs (also platelets or particles which are harder than RBCs) added to a suspension of deformable cells has been shown to be displaced toward the wall as a result of heterogeneous collisions between soft and stiffened cells, causing large displacements to stiff particles. ${ }^{33-35}$ All these studies have provided some important clues on the behavior of rigidified cells; however, it remains unclear how less deformable RBCs behave or distribute in dense, physiological suspensions, in which cell interactions are expected to be stronger. In particular, there is a scarcity of experimental data on the effect of RBC deformability on the velocity and hematocrit distributions of such suspensions in microscale flows that the present study aims to address.

Studies with denser, physiological RBC concentrations are not only important in understanding disease but also in improving our fundamental understanding of hydrodynamic interactions of deformable particles and providing microstructure information that is essential in constitutive modeling of such suspension flows, an area of considerable interest in the soft matter community. We have previously characterized the behavior of aggregating and nonaggregating healthy RBC flows in bifurcating microchannel geometries. These studies elucidated the strong coupling between the velocity field and the microstructure $e^{1-3,36,37}$ and, in particular, the role of $\mathrm{RBC}$ aggregation on partitioning at bifurcations, heterogeneity, and local viscosity. In the present study, we extend our previous work to probe the role of $\mathrm{RBC}$ deformability on the velocity and hematocrit distributions in microchannel flows. Hardened RBC suspensions perfused through straight microchannels at different hematocrits and flow rates are studied, and their behavior is compared to healthy RBC suspensions.

\section{EXPERIMENTAL METHODS}

\section{A. Sample preparation}

Human blood was collected from three different healthy donors with ethical approval from the South East London NHS Research Ethics Committee (Ref:10/H0804/21). RBCs were separated from whole blood via centrifugation and washed twice in phosphate buffered saline (PBS) before they were resuspended in PBS at hematocrits of $5 \%, 10 \%$, or $25 \%$. RBC hardening was carried out by incubating samples in glutaraldehyde (GA) solution of $0.08 \%$ for $15 \mathrm{~min}$, expected to decrease deformability approximately 3-4 fold. ${ }^{19,38}$ The samples were subsequently washed in PBS and resuspended at the desired hematocrit. Samples were adjusted to room temperature before experimentation. All experiments were carried out within the same day of venepuncture. Typical images of the samples studied are shown in Fig. 1(a). It should be noted that no significant difference in $\mathrm{RBC}$ morphology was observed between healthy and hardened samples.

\section{B. Microchannel fabrication}

All experiments were conducted in a straight, $16 \mathrm{~mm}$ long, microchannel geometry with a square cross-sectional area of $50 \mu \mathrm{m}$ 


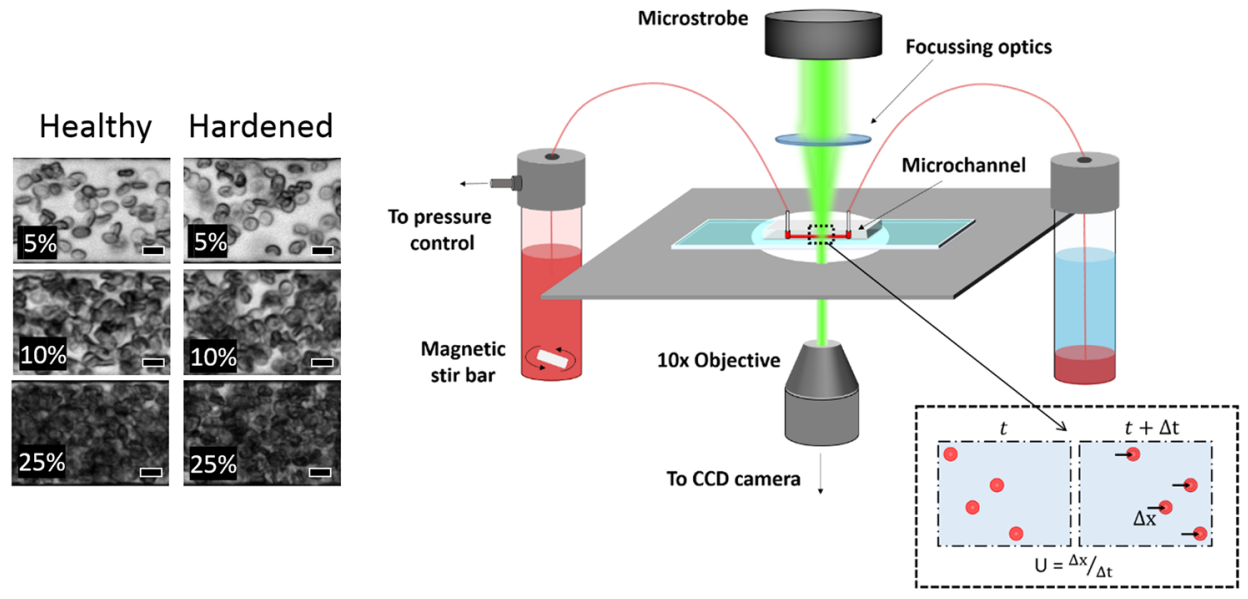

(a) (b)

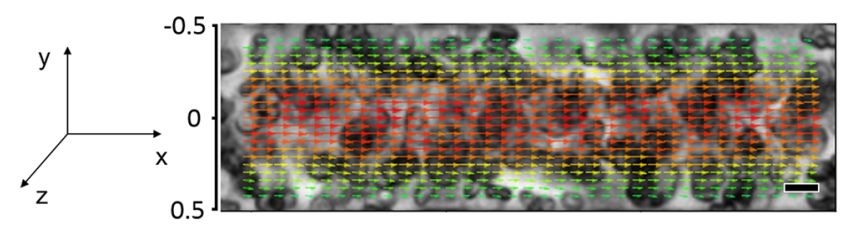

(c)
FIG. 1. (a) Sample images of healthy and hardened RBC samples of various hematocrits perfused through the microchannel at $45 \mathrm{~s}^{-1}$. (b) Schematic of the $\mu$ PIV experimental setup for brightfield imaging of microscale blood flow. (c) Sample vector plot obtained by PIV processing to preprocessed images. Multipass correlation methods were used with a vector spacing of $2.4 \mu \mathrm{m}$ ( 4 pixels). The first vector is positioned $6 \mu \mathrm{m}$ from the wall. The scale bar is $10 \mu \mathrm{m}$. The origin of the coordinate system was taken at the midplane of the channel and on its centerline so that the normalized radial coordinate, $y^{*}=y / W$, varied from -0.5 to 0.5 . width $(W)$. The microchannels were fabricated by standard soft lithography techniques. ${ }^{39}$ An SU-8 master was created and served as a negative mold. Sylgard 184 (Dow Corning) was used as PDMS prepolymer mixed with a curing agent at 10:1 ratio. The polymer mixture was degassed in a vacuum chamber to remove air bubbles, poured over the silicon mold, and baked at $65^{\circ} \mathrm{C}$ overnight. After curing, the PDMS replica was peeled off. Inlet and outlet perfusion holes were created using a biopsy punch, and subsequently, the PDMS chips were bonded onto glass slides using oxygen plasma treatment to both surfaces by a handheld corona device (BD$20 \mathrm{ACV}$, Rotaloc, Inc., USA). The origin of the coordinate system was taken at the midplane of the channel and on its centerline so that the normalized radial coordinate, $y^{*}=y / W$, varied from -0.5 to 0.5 .

\section{Experimental setup}

RBC solutions were perfused through the microchannels by a bespoke pneumatic microfluidic flow controller described previously. ${ }^{36}$ The volumetric flow rates were determined by integrating the measured velocity profiles-making use of quasi-3D assumptions as described in the work of Sherwood et al. ${ }^{2}$ (supplementary material, S3) - and were expressed in terms of a normalized velocity or nominal shear rate, $U^{*}=\bar{U} / W$, where $\bar{U}$ is the average flow velocity and $W$ is the width of the channel. $U^{*}$ varied from 1 to $250 \mathrm{~s}^{-1}$ in this study. RBC flows were imaged using a LabVIEW (National Instruments, USA) driven micro-Particle Image Velocimetry ( $\mu$ PIV) system described previously. ${ }^{1,2}$ The system, shown schematically in Fig. 1(b), comprises an epifluorescent inverted microscope (Leica DM ILM, Germany) equipped with a $10 \times$ objective $(\mathrm{NA}=0.25)$, a green Light Emitting Diode (LED) microstrobe illumination, and a Charged Coupled Device (CCD) camera (Hamamatsu, C8484-05C,
Japan). RBCs were used as flow tracers, and 60 pairs of images were acquired at a frame rate of $6 \mathrm{~Hz}$. The data acquisition time was $10 \mathrm{~s}$ and within this time frame, and the flow rate employed sedimentation can be considered negligible. ${ }^{40}$ A high flow rate (around $U^{*}$ $=650 \mathrm{~s}^{-1}$ ) was also applied between each measurement in order to redistribute the RBCs and minimize accumulated sedimentation effects. The time between image pairs $(d t)$ was adjusted between 0.5 and $4 \mathrm{~ms}$ depending on the flow rate; this was selected so that the $\mathrm{RBC}$ maximum displacement in the center of the flow was less than half of the interrogation window (IW) width.

The acquired images were preprocessed in MATLAB: images were cropped to $2 \mathrm{~W}$ and aligned and rescaled according to the PIV interrogation windows (IW) such that $W$ was an integer multiple of the IW width. The velocity field was determined by implementing multipass ensemble PIV cross-correlation algorithms in JPIV (www.vennemann-online.de/jpiv). Three passes with a final interrogation window size of $16 \times 8$ pixels and $50 \%$ overlap were applied. Vector validation was conducted using the normalized median test. Fewer than $0.1 \%$ of detected vectors were deemed invalid and were replaced by the median of the neighboring vectors. The image resolution after preprocessing was $0.60 \mu \mathrm{m} / \mathrm{pixel}$, and hence, the spatial resolution of the measurements (i.e., vector spacing) was $2.4 \mu \mathrm{m}$ (4 pixels). Due to diffraction, the pixels near the wall could not properly contribute to the PIV processing; hence, the first vector was located 10 pixels from the wall, i.e., $6 \mu \mathrm{m}$. For the more dilute sample (5\%), the first vectors considered were located $7.2 \mu \mathrm{m}$ away from the wall, in order to avoid PIV errors due to reduced RBC concentration near the wall.

Figure 1(c) shows a sample vector plot obtained. A region of interest (ROI), two channel widths long and located at channel midlength (i.e., $160 \mathrm{~W}$ downstream of the inlet), was used to 
estimate the velocity profiles across the channel. The choice of ROI was verified by (a) comparing velocity profiles produced from ROIs three and five channel widths long, and the differences in the measured velocity profiles were below $0.03 \%$ and $0.04 \%$, respectively, and (b) by comparing velocity profiles measured upstream and downstream of the ROI. The flow rates determined from these profiles were found to be consistent within $2 \%$, providing also an indication of the error in the velocity measurements.

It should be noted that in the current setup the depth of correlation is expected to be larger than the channel depth, ${ }^{41}$ and hence, the measured velocities are expected to be biased toward lower velocities due to the out of plane motion of RBCs. Poelma et al. ${ }^{42}$ suggested a correction method to account for the velocity underestimation due to correlation depth effects. While the velocity profiles are expressed in a normalized form and hence no such correction is necessary, the $U^{*}$ values have been adjusted by dividing the measured velocities by a factor of 0.66 as suggested by Sherwood et al. ${ }^{2}$ for the current setup.

\section{Velocity profiles}

Velocity profiles were extracted by averaging the measured vectors in the direction of the flow in the region of interest (ROI). The experimental data were fitted using the following equation: ${ }^{2}$

$$
U=u\left(\frac{\cosh \left(0.5^{m}\right)-\cosh \left(\left|y_{i}^{*}\right|^{m}\right)}{\cosh \left(0.5^{m}\right)-1}\right)+u_{0},
$$

where $u$ is a weighting parameter related to the average velocity, $u_{o}$ is the wall slip velocity due to the finite size of RBCs, $y_{i}{ }^{*}$ is the normalized radial coordinate ranging between -0.5 and 0.5 , and exponent $m$ is a fitting parameter indicating how blunt the velocity profile is. Equation (1) is an empirical fitting approach with $u, m$, and $u_{o}$ as free parameters, used to smooth the data and remove noise for the calculation of the flow rate; it is a simplified form of the analytical solution for the laminar flow velocity in a square microchannel [Eq. (3)], which accounts for the nonzero wall velocity at the wall and varying profile bluntness. Its derivation is described in detail in the work of Sherwood et al. ${ }^{2}$ (supplementary material, S3).

The shape of the velocity profiles is a measure of the nonNewtonian character of the flow as shear thinning fluids, like blood, are known to exhibit blunter velocity profiles. In this study, a bluntness index, expressed as the difference of the measured profile from the analytical one for a Newtonian fluid, was employed to facilitate a comparison of the velocity profiles obtained with RBCs of different degrees of deformability. This is defined as follows:

$$
B_{U}^{*}=\int_{-0.5}^{0.5}\left|\frac{u_{a}\left(y^{*}\right)}{\overline{u_{a}}}-\frac{u_{e}\left(y^{*}\right)}{\overline{u_{e}}}\right| d y^{*},
$$

where $y^{*}=y / W, u_{e}$ is the experimental velocity profile, $u_{a}$ is the analytical velocity profile for a Newtonian channel flow given by Bruus, ${ }^{43}$

$$
u_{a}\left(y^{*}\right)=\frac{4 h^{2} \Delta p}{\pi^{3} \mu L} \sum_{n, o d d}^{\infty} \frac{1}{n^{3}}\left[1-\frac{\cosh \left(n \pi \frac{y^{*}}{h}\right)}{\cosh \left(n \pi \frac{w}{2 h}\right)}\right],
$$

where $\Delta p$ is the pressure drop along length $\mathrm{L}$, and $\mu$ is the dynamic viscosity. Since $\overline{u_{a}}$ is proportional to $\Delta p / \mu L$ and results are presented in normalized form, these parameters do not need to be determined.

\section{E. Hematocrit profiles}

The hematocrit distribution was determined from the timeaveraged intensity of the acquired images as described in the work of Sherwood et al. ${ }^{2}$ A calibration procedure was first performed in which the average image intensity of perfused RBC solutions with feed hematocrits ranging between $5 \%$ and $40 \%$ was obtained. The image intensity was correlated with hematocrit using the expression ${ }^{2}$ (supplementary material, S2)

$$
H_{c}=\frac{1}{b} \ln \left(1-\frac{1-I^{*}}{a}\right)
$$

where $\mathrm{I}^{*}$ is the normalized image intensity defined as $I_{\text {raw }} / I_{\max }$, with $I_{\text {raw }}$ as the raw image intensity and $I_{\max }$ as the maximum image intensity in the absence of RBCs (i.e., measured outside of the channel). The calibration was conducted for both healthy and hardened cells since GA slightly alters the optical properties of the samples. The fitting parameters $a$ and $b$ were found to be equal to $0.72 \pm 0.03$ and $-9.1 \pm 0.5$ for the healthy and $0.77 \pm 0.02$ and $-9.7 \pm 0.4$ for the GA hardened samples, respectively.

Similarly to velocity, a hematocrit bluntness index was employed to characterize the shape of hematocrit profiles as described in the work of Sherwood et al. ${ }^{2}$ This is defined as

$$
B_{H}^{*}=\frac{3}{2}\left\{\frac{\int_{-0.5}^{0.5} H\left(y^{*}\right) d y^{*}-\int_{-0.166}^{0.166} H\left(y^{*}\right) d y^{*}}{\int_{-0.5}^{0.5} H\left(y^{*}\right) d y^{*}}\right\}
$$

and compares the hematocrit profile in the central third of the channel to the overall hematocrit distribution across the channel. A value of zero indicates that all RBCs flow within the central third of the channel, whereas a value of one indicates a uniform distribution.

\section{RESULTS}

\section{A. Velocity profiles}

Figures 2(a) and 2(b) show typical velocity profiles for $5 \%$ and $25 \%$ hematocrit suspensions of healthy and hardened (GA $0.08 \%$ ) RBCs from a single donor, respectively. The profiles are normalized by the average velocity $U_{\text {avg }}$ calculated from the two-dimensional (fitted) velocity profile. The wall slip velocity shown in the profiles corresponds to the $u_{0}$ term of Eq. (1). The analytical solution for Newtonian flow in the square microchannel ${ }^{43}$ is also indicated by the gray area. At low hematocrit (5\%), the velocity profiles of both healthy and hardened samples match and they both are close to the analytical profile as expected, given the low concentration of cells. On the contrary, when the hematocrit increases to $25 \%$ [Fig. 2(b)], healthy RBCs exhibit a blunt velocity profile, whereas the hardened ones exhibit a Newtonian-like behavior. Bluntness is a well-documented aspect of the velocity profiles of shear thinning fluids including those of healthy RBC suspensions. ${ }^{1,44,45}$ The decrease in bluntness observed with hardened cells is commensurate with the flow curves for hardened RBCs which show a noticeable decrease in shear thinning behavior with the loss of deformability. ${ }^{12,15}$ 


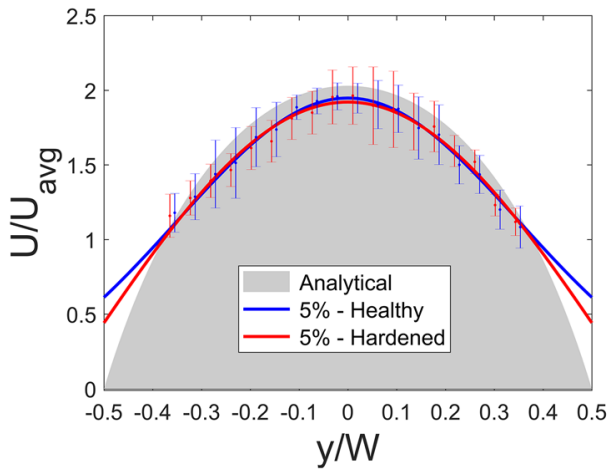

(a)

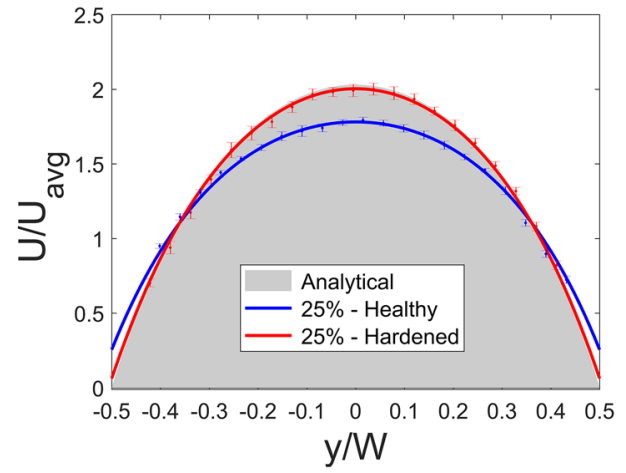

(b)
FIG. 2. Velocity profiles of healthy and hardened (GA 0.08\%) RBC samples from a single donor obtained in the square microchannel at (a) $5 \%$ and (b) $25 \%$ feed hematocrits. The gray area indicates the analytical velocity profile for a Newtonian fluid in a square channel [Eq. (3)]. Each experimental data point represents the average velocity from 23 velocity vectors measured in the axial direction within the ROI. Error bars correspond to the standard deviation of the measurements; the latter increase at the low hematocrit case due to the nature of the $\mu$ PIV technique.
In order to quantify the impact of impaired RBC deformability on the velocity distribution, the bluntness index defined in Eq. (2) was estimated for all measured velocity profiles. In Fig. 3, the bluntness index data are grouped in box plots in order to better illustrate the profile changes with hardening for hematocrits of $5 \%, 10 \%$, and $25 \%$.
Figure 3(a) indicates that at low hematocrit (5\%) the velocity profiles of healthy and hardened cells have similar bluntness, i.e., hardening has a small, almost negligible, effect on the shape of the profiles. As the hematocrit increases to $10 \%$ and $25 \%$, a progressive reduction in velocity bluntness with loss of deformability can be discerned. This becomes more apparent at higher hematocrit

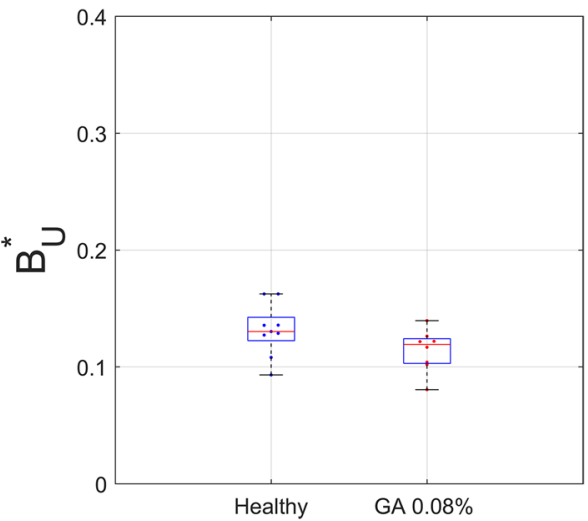

(a)

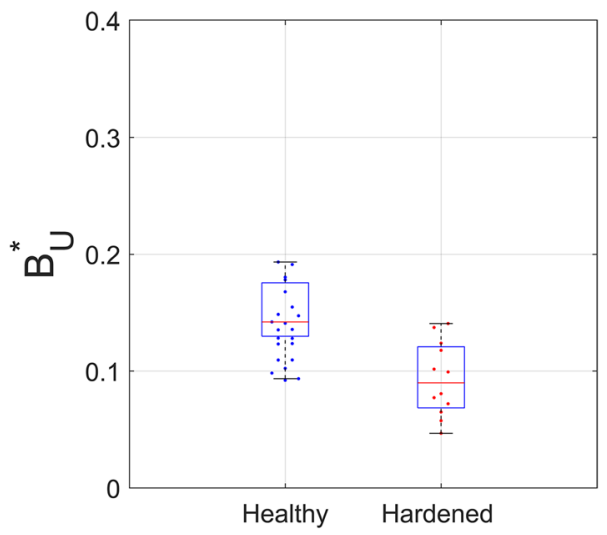

(b)
FIG. 3. Velocity bluntness index of the measured velocity profiles within the range of $1-250 \mathrm{~s}^{-1}$ for each of the samples at (a) $5 \%$, (b) $10 \%$, and (c) $25 \%$ feed hematocrits. The central red lines indicate the median of the measurements, and the bottom and top sides of the box indicate the 25 th and 75 th percentiles, respectively. 


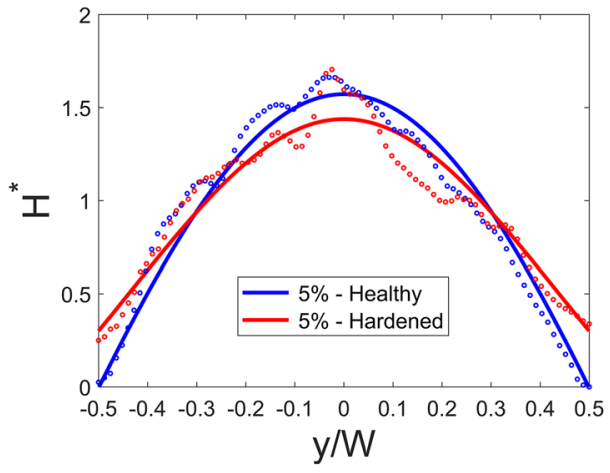

(a)

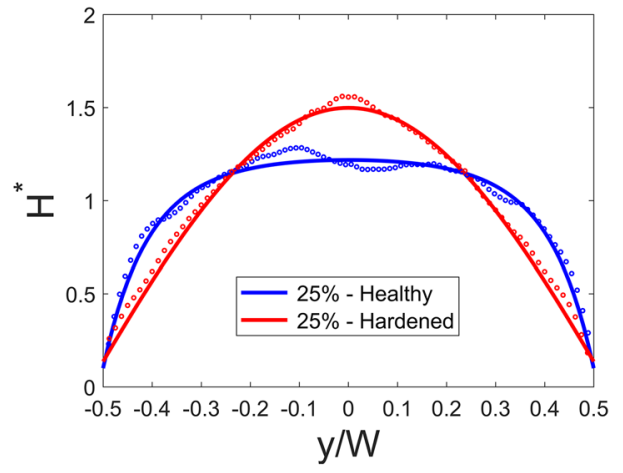

(b)
FIG. 4. Hematocrit profiles normalized by the average hematocrit for (a) $5 \%$ and (b) $25 \%$ feed hematocrits.
(25\%) [Fig. 3(c)]. The effect of hematocrit on bluntness is more pronounced for the healthy samples, whereas hardened samples appear to exhibit similar bluntness levels regardless of the feed hematocrit.

\section{B. Hematocrit distributions}

Figures 4(a) and 4(b) show typical hematocrit profiles determined from flows of healthy and hardened RBCs at 5\% and 25\% feed hematocrits. The fitted hematocrit profiles are shown along with the raw data and normalized by the average hematocrit obtained by integration of the hematocrit profiles across the channel. At low feed hematocrits (5\%), the distribution of RBCs is more uniform across the channel for both healthy and hardened samples during flow. At higher hematocrit (25\%), the healthy RBC profile appears more uniform in the central part of the channel in agreement with our previous work. ${ }^{2,36}$ However, hardened RBCs seem to

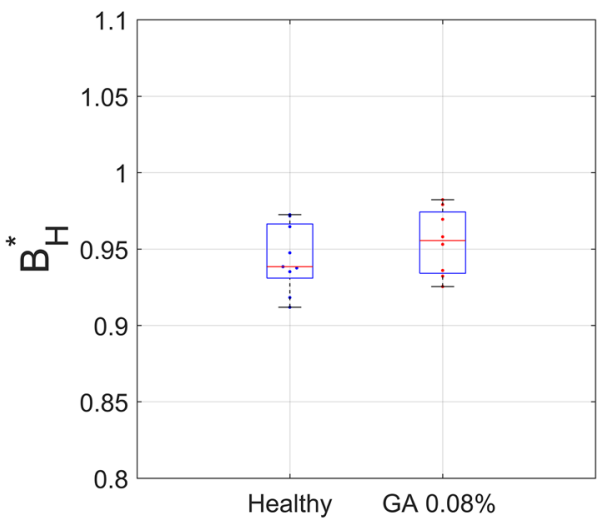

(a)

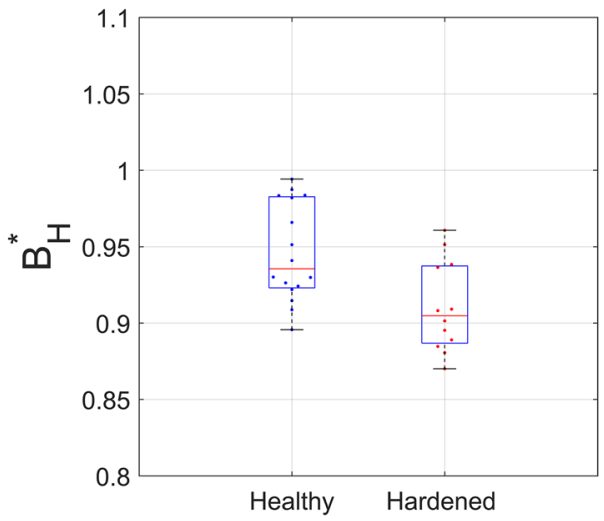

(b)

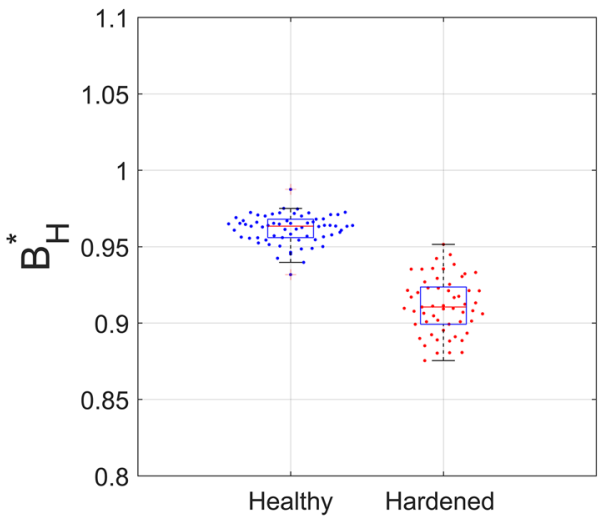

(c)
FIG. 5. Bluntness index of measured hematocrit profiles at feed hematocrits of (a) $5 \%$, (b) $10 \%$, and (c) $25 \%$. Red lines indicate the median and the box indicates the 25th and 75th percentiles, and outliers are plotted individually with "t" and correspond to the outer $0.7 \%$ of the data. 


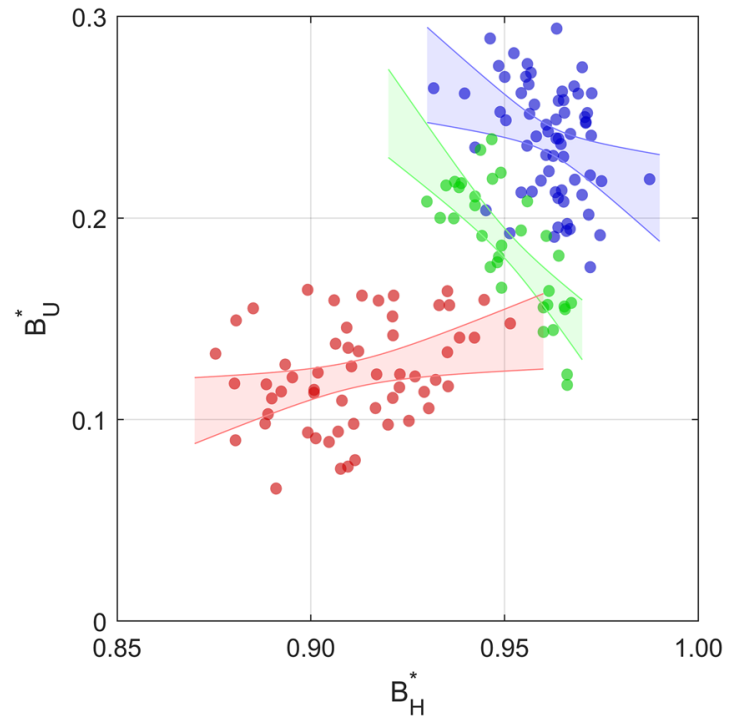

FIG. 6. Velocity vs hematocrit bluntness index for healthy and hardened RBC samples. Blue and green symbols indicate healthy RBC samples from the present study and Sherwood et al., ${ }^{2}$ respectively. Red symbols indicate hardened RBC samples from the present study. The feed hematocrit is $25 \%$, and data from three donors are shown for the present study. Shaded areas and bounding lines correspond to $95 \%$ confidence bounds on linear regression analysis. The corresponding statistics are provided in Table I.

concentrate in the center of the channel producing sharper hematocrit profiles.

The hematocrit profile bluntness was quantified using Eq. (5) for all measured hematocrit profiles and illustrated in Fig. 5 using box plots. Figure 5(a) shows that at 5\% hematocrit hardened RBCs exhibit on average blunter hematocrit distributions across the channel, consistent with reports of decreased migration of stiffer cells. On the contrary, the bluntness index of denser suspensions, Figs. 5(b) and 5(c), shows the opposite trend: a reduction in deformability is associated with a progressive decrease in hematocrit bluntness. This effect is more pronounced for the higher hematocrit case (25\%).

\section{Velocity-hematocrit bluntness correlation}

In order to better illustrate the effect of RBC deformability on the relationship between the velocity and hematocrit distributions, results obtained with RBCs from three different donors were collected and a correlation map between the estimated velocity and hematocrit bluntness indices was produced in Fig. 6 for the 25\%

TABLE I. Pearson correlation coefficients for velocity and hematocrit bluntness indices.

\begin{tabular}{lccc}
\hline \hline & $\begin{array}{c}\text { Healthy RBCs } \\
\text { (present study) }\end{array}$ & $\begin{array}{c}\text { Hardened RBCs } \\
\text { (present study) }\end{array}$ & $\begin{array}{c}\text { Healthy RBCs } \\
\text { (Sherwood } \text { et al. }{ }^{2} \text { ) }\end{array}$ \\
\hline $\mathrm{r}$ & -0.33 & 0.31 & $\begin{array}{l}-0.76 \\
<10^{-6}\end{array}$ \\
$\mathrm{p}$ & 0.006 & 0.019 & $<19$ \\
\hline \hline
\end{tabular}

hematocrit case. Data from the study of Sherwood et al. ${ }^{2}$ are also shown. The healthy RBC samples (blue) show a negative correlation between the velocity and hematocrit profile bluntness in agreement with our previous studies. ${ }^{2}$ Conversely, the hardened samples exhibit a positive correlation between hematocrit and velocity bluntness. The trends were found to be statistically significant using Pearson's correlation test (Table I).

\section{DISCUSSION}

The measured velocity profiles (Fig. 2) indicate that reduced deformability results in sharper, more Newtonian-like, velocity profiles in dense RBC suspensions [Figs. 2(b), 3(b), and 3(c)] but not dilute RBC flows [Figs. 2(a) and 3(a)]. This implies a reduction in the shear thinning nature of the suspensions with $\mathrm{RBC}$ hardening, which is well documented in the literature; Chien ${ }^{12}$ and Lanotte et al. ${ }^{15}$ demonstrated that reduction of RBC deformability suppresses shear thinning behavior as hardened RBCs preserve their hydrodynamic shape under shear.

The deformability of RBCs results in symmetry breaking in Stokes flow and a deformability induced lift force that causes the cells to migrate laterally, unlike rigid particles. $\mathrm{RBC}$ hardening decreases deformability-induced lift forces, and hence, hardened cells are expected to laterally migrate ${ }^{24,44}$ less than deformable ones, resulting in a more uniform distribution across the channel. This seems to be the case for the dilute RBC suspension (5\%) data in Figs. 4(a) and 5(a) that show a slight increase in the hematocrit profile bluntness for hardened RBCs compared to normal RBCs. This is in agreement with published works ${ }^{29}$ that reported wider RBC distributions in microscale flows of dilute suspensions of rigidified RBCs compared to normal ones.

On the contrary, the hematocrit findings for denser suspensions [Figs. 4(b), 5(b), and 5(c)] show sharper hematocrit profiles for hardened RBCs compared to healthy ones, implying that hardened RBCs concentrate more toward the center of the channel. This effect is more pronounced for the highest hematocrit case studied (25\%) and results in an inversion of the correlation between velocity and hematocrit profile bluntness, which is known to be negative for healthy RBCs but becomes positive for the hardened cells in this study. This finding is in contrast with in silico studies of $\mathrm{RBC}$ flows that have reported more dispersed distributions for less deformable cells. ${ }^{27,28,46}$ However, our results do bear similarity to the sharp concentration distributions reported for dense, rigid particle suspensions in rectangular channel flows by Lyon and Leal ${ }^{47}$ that were attributed to shear-induced migration effects. Although hardened RBCs are not expected to be fully rigid at the concentration of GA used, membrane stiffening affects both individual RBC motion and collision dynamics in RBC suspensions; these effects might combine with the altered local shear gradients in the measured flows to give rise to the observed concentration profiles. ${ }^{25,34}$ Wall induced lift forces are expected to be "screened" in the case of dense RBC suspensions ${ }^{25}$ which might explain the absence of any observed changes in hematocrit distribution for dense RBC suspensions with a change in viscosity contrast between cells and the suspending medium (hence $\mathrm{RBC}$ deformability) reported in the recent numerical studies by de Haan et al. ${ }^{48}$ and Saadat et al. ${ }^{49}$ However, the increased tumbling motion of the hardened cells is thought to enhance the collision rate between RBCs and the wall producing larger displacements and 
transverse velocities for RBC in the near wall region ${ }^{50}$ which could contribute to the hematocrit trends in the present study.

The inverted relationship between velocity and hematocrit bluntness is counterintuitive as, based on the dependency of suspension viscosity on particle concentration, one would expect a higher centerline hematocrit to correspond with increased local viscosity and a correspondingly blunter velocity profile, which is not the case for hardened cells in this study. This might imply that for the range of hematocrits examined, there is no significant effect of cell concentration on viscosity. Indeed, Chien ${ }^{11}$ reported very moderate increases in viscosity for hardened cells when the hematocrit varied from $0 \%$ to $30 \%$. Hardened RBCs are expected to be discoidal in shape, and hence, orientation effects with respect to the flow might become important. Simulations of platelike particles have shown that as the volume fraction increases, the particles tend to align in ordered layers forming stacks as there is not sufficient volume around the particles to rotate freely. ${ }^{51}$ Although GA hardened RBCs have been observed to exhibit increased tumbling motion which can lead to higher effective viscosity, at high cell concentrations flow alignment and ordering might take place, which could further explain the observed trends. An ordered, two file flow has also been shown by Shen et al. ${ }^{30}$ for RBC suspensions with high viscosity contrast, albeit for lower hematocrit flows.

The observed hematocrit distributions are expected to have an impact on partitioning of RBC suspensions in bifurcations and the downstream organization of the suspension, as has been demonstrated with aggregated RBC suspensions $s^{1-3,37,52}$ and also by the inversion of hematocrit partitioning in bifurcations found by Shen et al. ${ }^{30}$ for dilute suspensions of RBCs with altered deformation characteristics. The concentration of hardened RBCs toward the axis of the channels would likely enhance the Fåhraeus effect and plasma skimming, causing low flow rate vessels to receive fewer cells and high flow rate ones more, potentially making them more prone to jamming as cells are less deformable. This might enhance heterogeneity of both hematocrit and viscosity in the microcirculation and have implications for perfusion. Such hemodynamic changes could in part explain the various microangiopathies and adverse effects associated with pathologies known to impair the properties of RBCs or with blood transfusion. Deformability induced microhemodynamic changes might be exacerbated in the presence of plasma which has been recently shown to have viscoelastic properties $^{53,54}$ that can further impact migration dynamics and cell interactions.

\section{STUDY LIMITATIONS}

The velocity and hematocrit profile comparisons between healthy and hardened RBCs presented in Figs. 2 and 4 are based on sample(s) from the same donor for consistency and in order to avoid interdonor variation in RBC properties. Samples from 3 donors were used in the hematocrit vs velocity bluntness correlation in Fig. 6, as well as the data from the earlier study of Sherwood et $a$ l. $^{2}$ Both intrasample and interdonor variation in RBC deformability have been reported to be approximately $10 \% .{ }^{55,50}$ These variations are expected to have minimal effects in the distribution of RBCs in the channel ${ }^{31,32,35}$ and the differences observed between healthy and hardened RBCs, as the reported trends were consistent between donors.
Glutaraldehyde is commonly employed to artificially harden RBCs and has been found to perform better than other agents, such as diamide. ${ }^{14,56}$ Although it is an artificial treatment that does not entirely replicate $\mathrm{RBC}$ changes in disease, it provides a good starting point to appreciate the role of such changes in a systematic manner. In addition to altering RBC deformability, GA also induces changes to other cell properties, such as cell volume, ${ }^{57}$ osmolarity, ${ }^{58}$ and charge, ${ }^{59}$ which could potentially affect the cell dynamics and interactions. However, the differences between healthy and hardened samples found in the present study are expected to be primarily due to RBC deformability as the GA concentration used is well above the critical value $\left(0.003 \%{ }^{60}\right)$ required to produce statistically significant changes in the latter and changes vary almost linearly with GA concentration. ${ }^{19,38}$

Incubation with glutaraldehyde can induce cell clustering, which could give rise to increased migration and hence the sharper hematocrit profiles observed in Fig. 6. In order to rule out the possibility of GA induced RBC clustering, the intensity distribution of images of hardened and healthy RBCs was examined, a technique demonstrated in our previous work as able to discern suspensions of aggregating RBCs from those suspended in PBS. ${ }^{61}$ The two distributions were found to be statistically similar $(p=0.99)$ by means of Kolmogorov-Smirnov testing.

Finally, various factors influence the performance of the $\mu \mathrm{PIV}$ technique which have been discussed in detail in the work of Sherwood et al. ${ }^{2}$ In brief, the large depth of focus due to the microscopy setup and the relatively dense suspension of biconcave RBCs result in underestimating the velocity, therefore requiring a velocity correction according to Poelma et al. ${ }^{42}$

\section{CONCLUSIONS}

A series of experiments were conducted to probe the role of red blood cell deformability on microhemodynamics. Significant differences in the distribution of flow velocities and hematocrit were observed between suspensions of deformable (healthy) and less deformable (hardened) RBCs perfused through straight microchannels.

A reduction in velocity bluntness with loss of deformability was observed, and this trend was more apparent in the higher hematocrit cases examined. The effect of deformability on hematocrit distributions was found to be depended on the feed hematocrit. In the dilute case (5\%), less deformable RBCs produced more dispersed cell distributions, whereas for the denser case (25\%) the hardened cells were shown to concentrate more toward the centerline of the channel, creating sharper distributions compared to normal RBCs.

The findings illustrate that the suspension microstructure in microchannel flows is the result of a complex interplay between migration and cell-cell interactions that are both deformability and concentration dependent and may aid the development of theoretical frameworks to describe the microscale behavior of dense suspensions of deformable particles. The altered flow characteristics of suspensions of less deformable RBCs are expected to have an impact on RBC flow partitioning at bifurcations, increasing flow and hematocrit heterogeneity in the microcirculation with implications for tissue perfusion. Future work will investigate the impact of RBC deformability on flow partitioning in bifurcating microchannels extending our previous work with aggregating blood. ${ }^{1-3,37}$ 


\section{ACKNOWLEDGMENTS}

Support for this work from the UCL Doctoral Training Programme in Medical Device Innovation is gratefully acknowledged. This programme was funded by University College London (UCL), EPSRC, National Institute for Health Research (NIHR) Biomedical Research Centres at University College London Hospitals, Great Ormond Street Hospital, and Moorfields Eye Hospital NHS Foundation Trust and UCL Ophthalmology. Dr. Agrawal was supported by an Overseas Research Training Fellowship (Grant No. NMRC/002/2012) from the National Medical Research Council, Ministry of Health, Singapore. The views expressed are those of the author(s) and not necessarily those of the NHS, the NIHR, or the Department of Health.

\section{REFERENCES}

${ }^{1}$ J. M. Sherwood, E. Kaliviotis, J. Dusting, and S. H. Balabani, "Viscosity and velocity distributions of aggregating and non-aggregating blood in a bifurcating microchannel," Biomech. Model. Mechanobiol. 13, 259-273 (2014).

${ }^{2}$ J. M. Sherwood, D. Holmes, E. Kaliviotis, and S. Balabani, "Spatial distributions of red blood cells significantly alter local hemodynamics," PLoS One 9, e100473 (2014).

${ }^{3}$ E. Kaliviotis, J. M. Sherwood, and S. Balabani, "Local viscosity distribution in bifurcating microfluidic blood flows," Phys. Fluids 30, 030706 (2018).

${ }^{4}$ D. K. Kaul and R. L. Nagel, "Sickle cell vasoocclusion: Many issues and some answers," Experientia 49, 5-15 (1993).

${ }^{5}$ N. Mohandas and P. G. Gallagher, "Red cell membrane: Past, present, and future," Blood 112, 3939-3948 (2008).

${ }^{6}$ X. Li, E. Du, H. Lei, Y.-H. Tang, M. Dao, S. Suresh, and G. E. Karniadakis, "Patient-specific blood rheology in sickle-cell anaemia," Interface Focus 6, 20150065 (2016).

${ }^{7} \mathrm{M}$. Fornal et al., "Erythrocyte stiffness in diabetes mellitus studied with atomic force microscope," Clin. Hemorheol. Microcirc. 35, 273-276 (2006).

${ }^{8} \mathrm{~K}$. Tsukada, E. Sekizuka, C. Oshio, and H. Minamitani, "Direct measurement of erythrocyte deformability in diabetes mellitus with a transparent microchannel capillary model and high-speed video camera system," Microvasc. Res. 61, 231-239 (2001).

${ }^{9}$ S. Shin, Y. Ku, N. Babu, and M. Singh, "Erythrocyte deformability and its variation in diabetes mellitus," Indian J. Exp. Biol. 45(1), 121-128 (2007).

${ }^{10} \mathrm{R}$. Agrawal et al., "Assessment of red blood cell deformability in type 2 diabetes mellitus and diabetic retinopathy by dual optical tweezers stretching technique," Sci. Rep. 6, 15873 (2016)

${ }^{11}$ S. Chien, S. Usami, R. J. Dellenback, and M. I. Gregersen, "Blood viscosity: Influence of erythrocyte deformation," Science 157, 827 (1967).

${ }^{12} \mathrm{~S}$. Chien, "Shear dependence of effective cell volume as a determinant of blood viscosity," Science 168, 977-979 (1970).

${ }^{13}$ S. Chien, "Red cell deformability and its relevance to blood flow," Annu. Rev. Physiol. 49, 177-192 (1987).

${ }^{14}$ A. M. Forsyth, J. Wan, W. D. Ristenpart, and H. A. Stone, "The dynamic behavior of chemically 'stiffened' red blood cells in microchannel flows," Microvasc. Res. 80, 37-43 (2010).

${ }^{15} \mathrm{~L}$. Lanotte et al., "Red cells' dynamic morphologies govern blood shear thinning under microcirculatory flow conditions," Proc. Natl. Acad. Sci. U. S. A. 113, 13289-13294 (2016).

${ }^{16}$ S. Chien et al., "Rheology of sickle cells and its role in microcirculatory dynamics," Prog. Clin. Biol. Res. 240, 151-165 (1987).

${ }^{17}$ G. A. Pantely et al., "Increased vascular resistance due to a reduction in red cell deformability in the isolated hind limb of swine," Microvasc. Res. 35, 86-100 (1988).

${ }^{18} \mathrm{P}$. Cabrales, "Effects of erythrocyte flexibility on microvascular perfusion and oxygenation during acute anemia," Am. J. Physiol.-Heart Circ. Physiol. 293, H1206-1215 (2007).
${ }^{19}$ J. M. Sosa, N. D. Nielsen, S. M. Vignes, T. G. Chen, and S. S. Shevkoplyas, "The relationship between red blood cell deformability metrics and perfusion of an artificial microvascular network," Clin. Hemorheol. Microcirc. 57, 275-289 (2014).

${ }^{20} \mathrm{~J}$. M. Burns et al., "Deterioration of red blood cell mechanical properties is reduced in anaerobic storage," Blood Transfus. 14, 80-88 (2016).

${ }^{21} \mathrm{D}$. K. Wood et al., "A biophysical indicator of vaso-occlusive risk in sickle cell disease," Sci. Transl. Med. 4, 123 ra26 (2012).

${ }^{22} \mathrm{~J}$. Dupire, M. Socol, and A. Viallat, "Full dynamics of a red blood cell in shear flow," Proc. Natl. Acad. Sci. U. S. A. 109, 20808-20813 (2012).

${ }^{23} \mathrm{H}$. L. Goldsmith and J. Marlow, "Flow behaviour of erythrocytes. I. Rotation and deformation in dilute suspensions," Proc. R. Soc. London, Ser. B 182, 351-384 (1972).

${ }^{24}$ S. Losserand, G. Coupier, and T. Podgorski, "Migration velocity of red blood cells in microchannels," Microvasc. Res. 124, 30-36 (2019).

${ }^{25} \mathrm{X}$. Grandchamp, G. Coupier, A. Srivastav, C. Minetti, and T. Podgorski, "Lift and down-gradient shear-induced diffusion in red blood cell suspensions," Phys. Rev. Lett. 110, 108101 (2013).

${ }^{26}$ D. A. Fedosov, B. Caswell, S. Suresh, and G. E. Karniadakis, "Quantifying the biophysical characteristics of Plasmodium-falciparum-parasitized red blood cells in microcirculation," Proc. Natl. Acad. Sci. U. S. A. 108, 35-39 (2011).

${ }^{27}$ J. Zhang, P. C. Johnson, and A. S. Popel, "Effects of erythrocyte deformability and aggregation on the cell free layer and apparent viscosity of microscopic blood flows," Microvasc. Res. 77, 265-272 (2009).

${ }^{28} \mathrm{P}$. Bagchi, "Mesoscale simulation of blood flow in small vessels," Biophys. J. 92, 1858-1877 (2007).

${ }^{29}$ T. Sasaki, J. Seki, T. Itano, and M. Sugihara-Seki, “Cross-sectional distributions of normal and abnormal red blood cells in capillary tubes determined by a new technique," Biorheology 54, 153-165 (2018).

${ }^{30} \mathrm{Z}$. Shen et al., "Inversion of hematocrit partition at microfluidic bifurcations," Microvasc. Res. 105, 40-46 (2016).

${ }^{31}$ B. Namgung, Y. C. Ng, H. L. Leo, J. M. Rifkind, and S. Kim, "Near-wall migration dynamics of erythrocytes in vivo: Effects of cell deformability and arteriolar bifurcation," Front. Physiol. 8, 963 (2017).

${ }^{32} \mathrm{Y}$. Chen et al., "Margination of stiffened red blood cells regulated by vessel geometry," Sci. Rep. 7, 15253 (2017).

${ }^{33}$ H. Zhao, E. S. G. Shaqfeh, and V. Narsimhan, "Shear-induced particle migration and margination in a cellular suspension," Phys. Fluids 24, 011902 (2012).

${ }^{34} \mathrm{G}$. Závodszky et al., "Red blood cell and platelet diffusivity and margination in the presence of cross-stream gradients in blood flows," Phys. Fluids 31, 031903 (2019).

${ }^{35}$ A. Kumar and M. D. Graham, "Mechanism of margination in confined flows of blood and other multicomponent suspensions," Phys. Rev. Lett. 109, 108102 (2012).

${ }^{36}$ J. M. Sherwood, J. Dusting, E. Kaliviotis, and S. Balabani, "The effect of red blood cell aggregation on velocity and cell-depleted layer characteristics of blood in a bifurcating microchannel," Biomicrofluidics 6, 024119 (2012).

${ }^{37}$ E. Kaliviotis, J. M. Sherwood, and S. Balabani, "Partitioning of red blood cell aggregates in bifurcating microscale flows," Sci. Rep. 7, 44563 (2017).

${ }^{38} \mathrm{X}$. Liu et al., "The measurement of shear modulus and membrane surface viscosity of RBC membrane with Ektacytometry: A new technique," Math. Biosci. 209, 190-204 (2007).

${ }^{39}$ D. C. Duffy, J. C. McDonald, O. J. A. Schueller, and G. M. Whitesides, "Rapid prototyping of microfluidic systems in poly(dimethylsiloxane)," Am. Chem. Soc. 70, 4974-4984 (1998).

${ }^{40}$ C. Alonso, A. R. Pries, O. Kiesslich, D. Lerche, and P. Gaehtgens, "Transient rheological behavior of blood in low-shear tube flow: Velocity profiles and effective viscosity,” Am. J. Physiol. 268, H25-H32 (1995).

${ }^{41}$ M. G. Olsen and R. J. Adrian, "Out-of-focus effects on particle image visibility and correlation in microscopic particle image velocimetry," Exp. Fluids 29, S166-S174 (2000).

${ }^{42}$ C. Poelma, A. Kloosterman, B. P. Hierck, and J. Westerweel, "Accurate blood flow measurements: Are artificial tracers necessary?," PLoS One 7, e45247 (2012).

${ }^{43} \mathrm{H}$. Bruus, Theoretical Microfluidics (Oxford University Press, USA, 2008), p. 346 . 
${ }^{44}$ H. L. Goldsmith, “The microrheology of red blood cell suspensions," J. Gen. Physiol. 52, 5-28 (1968).

${ }^{45}$ J. J. Bishop, P. R. Nance, A. S. Popel, M. Intaglietta, and P. C. Johnson, "Effect of erythrocyte aggregation on velocity profiles in venules," Am. J. Physiol.-Heart Circ. Physiol. 280, H222-H236 (2001).

${ }^{46}$ D. Fedosov, "Multiscale modeling of blood flow and soft matter," Ph.D. thesis, Brown University, 2010.

${ }^{47}$ M. K. Lyon and L. G. Leal, "An experimental study of the motion of concentrated suspensions in two-dimensional channel flow. Part 1. Monodisperse systems," J. Fluid Mech. 363, 25-56 (1998).

${ }^{48} \mathrm{M}$. de Haan et al., "Numerical investigation of the effects of red blood cell cytoplasmic viscosity contrasts on single cell and bulk transport behaviour," Appl. Sci. 8, 1616 (2018)

${ }^{49}$ A. Saadat, C. J. Guido, and E. S. G. Shaqfeh, "Effect of cytoplasmic viscosity on red blood cell migration in small arteriole-level confinements," bioRxiv: 10.1101/572933 (2019).

${ }^{50}$ K. Tatsumi, S. Noguchi, A. Tatsumi, R. Kuriyama, and K. Nakabe, "Particle and red blood cell concentration distributions in narrow microchannel flows," Phys. Fluids 31(8), 082006 (2019).

${ }^{51} \mathrm{Q}$. Meng and J. J. L. Higdon, "Large scale dynamic simulation of plate-like particle suspensions. Part II: Brownian simulation," J. Rheol. 52, 37-65 (2008).

${ }^{52}$ E. Kaliviotis, D. Pasias, J. M. Sherwood, and S. Balabani, "Red blood cell aggregate flux in a bifurcating microchannel," Med. Eng. Phys. 48, 23-30 (2017).
${ }^{53}$ M. Brust et al., "Rheology of human blood plasma: Viscoelastic versus Newtonian behavior," Phys. Rev. Lett. 110, 078305 (2013).

${ }^{54}$ S. Varchanis, Y. Dimakopoulos, C. Wagner, and J. Tsamopoulos, "How viscoelastic is human blood plasma?," Soft Matter 14, 4238-4251 (2018).

${ }^{55} \mathrm{~J}$. G. G. Dobbe, G. J. Streekstra, M. R. Hardeman, C. Ince, and C. A. Grimbergen, "Measurement of the distribution of red blood cell deformability using an automated rheoscope," Cytometry 50, 313-325 (2002).

${ }^{56} \mathrm{H}$. Schmid-Schönbein and P. Gaehtgens, "What is red cell deformability?," Scand. J. Clin. Lab. Invest. Suppl. 156, 13-26 (1981).

${ }^{57}$ C. A. Squier, J. S. Hart, and A. Churchland, "Changes in red blood cell volume on fixation in glutaraldehyde solutions," Histochemistry 48, 7-16 (1976).

${ }^{58}$ F. M. Morel, R. F. Baker, and H. Wayland, "Quantitation of human red blood cell fixation by glutaraldehyde," J. Cell Biol. 48, 91-100 (1971).

${ }^{59}$ P. S. Vassar, J. M. Hards, D. E. Brooks, B. Hagenberger, and G. V. Seaman, "Physicochemical effects of aldehydes on the human erythrocyte," J. Cell Biol. 53, 809-818 (1972).

${ }^{60} \mathrm{O}$. K. Baskurt et al., "Comparison of three commercially available ektacytometers with different shearing geometries," Biorheology 46, 251-264 (2009).

${ }^{61}$ E. Kaliviotis, J. Dusting, J. M. Sherwood, and S. Balabani, "Quantifying local characteristics of velocity, aggregation and hematocrit of human erythrocytes in a microchannel flow," Clin. Hemorheol. Microcirc. 63, 123-148 (2016). 